\title{
MACHINE LEARNING APPLICATIONS IN IOT BASED AGRICULTURE AND SMART FARMING: A REVIEW
}

\author{
M.W.P Maduranga \\ IIC University of Technology \\ No 650, NR2, Phnom Penh, Cambodia
}

\author{
Ruvan Abeysekera \\ IIC University of Technology \\ No 650, NR2, Phnom Penh, Cambodia
}

\begin{abstract}
Internet of Things (IoT) technology has revolutionized every aspect of everyday life by making everything smarter. Among the vast range of IoT applications, IoT based smart agriculture has fascinated many researchers and has used Machine Learning(ML) and IoT technologies to conduct innovative researches. IoT based data-driven farm management techniques can help increase agricultural yields by planning input costs, reducing losses, and using resources more efficiently. The IoT generates big amount data with different characteristics based on location and time. To improve productivity of agriculture through intelligent farm management, the data analyzing must be well analyzed and processed. High-performance computing capability in ML opens up new opportunities for data-intensive science as the amount of data collected increases; ML algorithms could be applied to further enhance application intelligence and functionality. In this article we review existing approaches have been made to the smart agriculture and farming based on IoT and ML separately. Also we propose novel concepts that how can ML-IoT can be blended in such applications.
\end{abstract}

Keywords - Internet of Things (IoT), Machine Learning (ML), Machine Learning in IoT(MLIoT), Smart Agriculture, Smart Farming.

\section{INTRODUCTION}

The Internet of Things focuses on automating processes by reducing human-human interaction. In the automation process, the IoT uses electronic sensors to collect data, the controller processes the data, and the actuators complete the automation process. The focus of the IoT in agriculture is to automate all aspects of agriculture and agricultural methods to make the process quite efficient. Traditional methods of yield management, livestock management, weed management, soil management, water management, plant management and animal tracking are not fully automated and inefficient, including increased human interaction, increased labor costs and increased power consumption. Many researchers have focused on intelligent systems that monitor and control agricultural parameters by increasing productivity and efficiency. Intelligent systems collect data for measurements and get accurate results which can take the appropriate action. Current uses of smart farming systems include collecting data on environmental parameters such as soil moisture level, humidity, temperature, and $\mathrm{pH}$ levels. ML is a new way of computing intelligence using machines. A vast range of $\mathrm{ML}$ applications are also exist on smart agriculture and farming. Yield prediction, water management, live stock management are the few example for application of ML in this field. Its observed that there are various researches have been done for smart agriculture in IoT and ML individually. In this research we review those solutions and propose how ML and IoT blend for better and precision agriculture [1] [2] [3].

\section{AGRICULTURAL IOT}

Structures for IoT based agriculture have been introduced in several research papers [4] [5]. In generally, in an IoT solution, each sensor mote includes a microcontroller, multiple types of sensors (from simple temperature sensors to cameras), actuators, and wireless interfaces. These wireless interfaces could be WiFi, LoRaWAN, Zigbee etc. A local WSN gateway sends data through an Internet gateway and forms the network layer. To understand the data collected by the service tier, you need to perform data processing such as data visualization, data analysis, data storage, and protection. Ultimately, the application layer is the most important part here, allowing end users to control and monitor various processes in the farm and make important decisions based on forecasts, market trends. IoT-based agriculture has resulted in a variety of data generated from various sources on and around agricultural farms, in the form of voltage values to images, actuator states to robot positions. Quality data leads to quality and accurate information. Without having quality information, you won't be able to create predictive models using ML algorithms. Applying ML algorithms to these improved datasets allows for better analysis and accurate predictions. The IoT can easily collect and manage large amounts of data collected from sensors and integrate cloud computing services such as agricultural maps and cloud storage. Real-time data access from anywhere, anytime, enabling real-time monitoring and end-to-end connectivity between all parties [6] 


\section{International Journal of Engineering Applied Sciences and Technology, 2020 \\ Vol. 4, Issue 12, ISSN No. 2455-2143, Pages 24-27 \\ Published Online April 2020 in IJEAST (http://www.ijeast.com)}

\section{MACHINE LEARNING APPLICATIONS IN IOT BASED AGRICULTURE}

Machine learning (ML) could be consider as a novel way for machines to simulate humans learning activities, gain new knowledge, continually improve performance, and achieve unique maturity. In past few years, ML has been very successful in algorithms, theories, and applications, combined with other agricultural techniques to minimize crop costs and maximize yield. ML applications on agricultural farms can be widely used in areas such as disease detection, crop detection, irrigation planning, soil conditions, weed detection, crop quality, and weather forecasting. ML for analyzing the freshness of produce can be found after harvest (Freshness of fruits and vegetables), Shelf life, Product quality, Market analysis etc. ML application in IoT based agriculture could be based on main ML algorithms such as support vector machine (SVM), naive Bayes, discriminant analysis, K-nearest neighbor, , K-means clustering, fuzzy clustering, Gaussian mixture models, artificial neural networks (ANN), decision making and deep learning [7],[8],[9].[10].

\section{A. Plant Management}

Hybrid of ML and the IoT provides appropriate and controllable environment for growing crops through greenhouse technology. However, the spatio-temporal variability of crop growth environmental parameters and their mutual effects in protected agriculture make it difficult for traditional agriculture and environmental regulations to adapt to the growth of different types of plants at different stages of growth. Therefore, higher accuracy is needed from a monitoring and control perspective. Many works existing on designing and testing types of monitoring and control systems for adjusting temperature and humidity, brightness, $\mathrm{CO} 2$ concentration and other environmental parameters for the Internet of Things, technical and economic results. It is proposed that controlling the environment conditions for a specific type of plant can be controlled through IoT, sensors and actuators. Here the rules of controlling condition can done according to a Artificial Neural Network(ANN) setup at IoT cloud. [11] [12] [13]

\section{B. Crop and Yield Management}

ML based yield mapping could apply in farms based on collected data over IoT network through yield monitoring connected through GPS. The collected date which reveals the yield details will be mapped based on the types of farm land. Apart from that, ML systems together with IoT can use to predict and improve the yields in agriculture. Farmers rely mainly on agricultural experts to make decisions. Farmers and others use these systems without any knowledge of computer use. ML system can be used for crop production. This is a knowledge building system that generates information using existing knowledge. This enables farmers to make economically sound crop management decisions. Various such systems have been developed in view of the success of expert systems. The Internet of Things plays an important role in agriculture. Related works shows that ML systems can be built on the IoT and can make recommendations on the use of input data collected in real time [14] [15]

\section{Soil Management}

Several ML-based approaches can be applied to soil management. Soil data can be collected from wireless sensor nodes deployed on site. Then, collected data can be fed into ML algorithms to predict and analyze soil properties or classify the types of soil using supervised ML algorithms. Moreover most commonly used ML algorithms, K-nearest neighbor, support vector regression (SVR), Naive Bayes, etc. can be use to predict soil dryness based on precipitation and evaporative hydrology data [16] [17] [18]

\section{Diseases Management}

Blended ML and IoT can be used to identify and manage diseases in agricultural fields. ML methods further stimulate appropriate pesticides to protect crops from these infections and reduce labor. Such system assists producers by obtaining statistics and planning fertilizers, pesticides and irrigation accordingly. By accurately identifying the disease and providing accurate pesticide application and irrigation schemes, grape visibility and volume have been increased and extreme pesticide use reduced. Furthermore, architecture with deep learning methods for identifying and classifying speech steps of various plants. The audio steps at these factories are based on real-time captured visual information and travel through different areas of the farm via IoT-based camera sensor nodes deployed in crop field [19] [20].

\section{E. Weed Management}

Weed management is essentials for any farming. Weed mapping through ML has been investigated [21] [22]. To optimize this, we propose unmanned flying machine to take images and map weed in a field. Where flying machine can be control through an IoT network. Advanced IoT technologies such as NB-IoT can use to handle and manipulate large amount of data.

\section{F. Water Management}

Several systems have been implement on controlling water supply for a agriculture field as well as analyzing the water quality using ML [23] [24]. It can be develop intelligent systems that detect ground parameters such as soil moisture, soil temperature, and environmental conditions using IoT sensors. Then, use the same data to predict outdoor relative humidity. In addition, we can use hybrid machine learning and 


\section{International Journal of Engineering Applied Sciences and Technology, 2020 \\ Vol. 4, Issue 12, ISSN No. 2455-2143, Pages 24-27 \\ Published Online April 2020 in IJEAST (http://www.ijeast.com)}

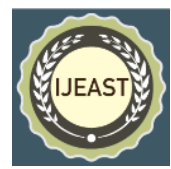

IoT systems to control water temperature and adjust to ambient temperature in intelligent way.

\section{G. Animal Tracking}

Animal tracking of an agriculture field is really important. Several researches have been made on tracking animals using IoT based sensors, and separate researches are carried on animal type classification [25] [26]. Together IoT and ML solution could solve this problem efficiency. Sensing the presence of an animal can be detected through IoT sensors. Traced animal can be classified and or else can study their living patterns, movements using ML techniques

\section{SUMMERY}

IoT-ML based agriculture is the next evolutional thing in smart agricultural and smart farming. Applying ML algorithms to data generated from various inputs from farms with the help of the agricultural IoT can make the system smarter and provide definitive information and make predictions. In this study, we analyze existing ML applications in agriculture, from process to results, each with its own strengths and weaknesses. Later, because most ML applications needed real-time data to train predictive algorithms, suggestions were made to implement new applications on the IoT. Farm management systems are evolving into reality by applying machine learning to sensor data Artificial intelligence (AI) system that provides richer suggestions and insights for subsequent work decisions and actions with a range of final production improvements. In the future, this range will be expect and enable wider use of ML models.

\section{REFERENCES}

[1] M. Popa, O. Prostean,and A.S. Popa, (2019) Machine Learning Approach for Agricultural IoT In Proc. International Journal of Recent Technology and Engineering (IJRTE),(pp 22-29)

[2] Medela, et al. (2013 )IoT Multiplatform networking to monitor and control wineries and vineyards. In: Future Network and Mobile Summit," IEEE,( pp. 1-10)

[3] Mohammad Saeid Mahdavinejad, et al (2018) Machine learning for internet of things data analysis: a survey in proc. Digital Communications and Network, (pp, 161175)

[4] Andreas Kamilaris, et al.(2016) Agri-IoT: A Semantic Framework for Internet of Things-enabled Smart Farming Applications," European Union.

[5] Prem Prakash Jayaraman, et al., (2018) Internet of Things Platform for Smart Farming: Experiences and Lessons Learnt,in proc Sensors, (pp 16, 1884)

[6] Castelli, Mauro, et al.,(2018) Supervised Learning: Classification, Reference Module in Life Sciences, in proc. Elsevier.
[7] R. L. F. Cunha, B. Silva and M. A. S. Netto,(2018) A Scalable Machine Learning System for Pre-Season Agriculture Yield Forecast," 2018 IEEE 14th International Conference on e-Science (e-Science), Amsterdam, 2018, (pp. 423-430).

[8] S. Dimitriadis and C. Goumopoulos, (2008)"Applying Machine Learning to Extract New Knowledge in Precision Agriculture Applications," 2008 Panhellenic Conference on Informatics, Samos, 2008, (pp. 100-104).

[9] T. Siddique, D. Barua, Z. Ferdous and A. Chakrabarty, "Automated farming prediction,(2017 ) Intelligent Systems Conference (IntelliSys), London, 2017, (pp. 757763).

[10] M. T. Shakoor, K. Rahman, S. N. Rayta and A. Chakrabarty, 2017 Agricultural production output prediction using Supervised Machine Learning techniques, 1st International Conference on Next Generation Computing Applications (NextComp), Mauritius, ( pp. 182-187).

[11] M. V. Ramesh et al. (2017), "Water quality monitoring and waste management using IoT," 2017 IEEE Global Humanitarian Technology Conference (GHTC), San Jose, CA, (pp. 1-7)

[12] C. J. G. Aliac and E. Maravillas,(2018) "IOT Hydroponics Management System," 2018 IEEE 10th International Conference on Humanoid, Nanotechnology, Information Technology,Communication and Control, Environment and Management (HNICEM), Baguio City, Philippines, (pp. 1-5).

[13] R. N. Rao and B. Sridhar,(2018) "IoT based smart cropfield monitoring and automation irrigation system," 2018 2nd International Conference on Inventive Systems and Control (ICISC), Coimbatore, 2018, pp. 478-483.

[14] A. A. Araby et al., "Smart IoT Monitoring System for Agriculture with Predictive Analysis," 2019 8th International Conference on Modern Circuits and Systems Technologies (MOCAST), Thessaloniki, Greece, 2019,( pp. 1-4)

[15] S. Dimitriadis and C. Goumopoulos, (2008)Applying Machine Learning to Extract New Knowledge in Precision Agriculture Applications," 2008 Panhellenic Conference on Informatics, Samos, ( pp. 100-104)

[16] O. Pandithurai, S. Aishwarya, B. Aparna and K. Kavitha,(2017) "Agro-tech: A digital model for monitoring soil and crops using internet of things (IOT)," In proc. 2017 Third International Conference on Science Technology Engineering \& Management (ICONSTEM), Chennai, (pp. 342-346)

[17] N. Ananthi, J. Divya, M. Divya and V. Janani,(2017) IoT based smart soil monitoring system for agricultural production," 2017 IEEE Technological Innovations in ICT for Agriculture and Rural Development (TIAR), Chennai, (pp. 209-214) 
[18] S. Athani, C. H. Tejeshwar, M. M. Patil, P. Patil and R. Kulkarni, (2017) Soil moisture monitoring using IoT enabled arduino sensors with neural networks for improving soil management for farmers and predict seasonal rainfall for planning future harvest in North Karnataka - India," 2017 International Conference on ISMAC (IoT in Social, Mobile, Analytics and Cloud) (ISMAC), Palladam, ( pp. 43-48)

[19] A. Adedoja, P. A. Owolawi and T. Mapayi, (2019) Deep Learning Based on NASNet for Plant Disease Recognition Using Leave Images," 2019 International Conference on Advances in Big Data, Computing and Data Communication Systems (icABCD), Winterton, South Africa, ( pp. 1-5).

[20] N. Materne and M. Inoue,(2018) IoT Monitoring System for Early Detection of Agricultural Pests and Diseases," 2018 12th South East Asian Technical University Consortium (SEATUC), Yogyakarta, Indonesia, (pp. 1-5).

[21] M. Pérez-Ortiz, P. A. Gutiérrez, J. M. Peña, J. TorresSánchez, F. López-Granados and C. Hervás-Martínez, "Machine learning paradigms for weed mapping via unmanned aerial vehicles," 2016 IEEE Symposium Series on Computational Intelligence (SSCI), Athens, 2016, pp. $1-8$.

[22] [22] S. T., S. T., S. G. G.S., S. S. and R. Kumaraswamy, "Performance Comparison of Weed Detection Algorithms," 2019 International Conference on Communication and Signal Processing (ICCSP), Chennai, India, 2019, pp. 0843-0847.

[23] [23] Y. Khan and C. S. See, "Predicting and analyzing water quality using Machine Learning: A comprehensive model," 2016 IEEE Long Island Systems, Applications and Technology Conference (LISAT), Farmingdale, NY, 2016, pp. 1-6.

[24] [24] M. R. Machado, T. R. Júnior, M. R. Silva and J. B. Martins, "Smart Water Management System using the Microcontroller ZR16S08 as IoT Solution," 2019 IEEE 10th Latin American Symposium on Circuits \& Systems (LASCAS), Armenia, Colombia, 2019, pp. 169-172.

[25] [25]L. Nóbrega, A. Tavares, A. Cardoso and P. Gonçalves, "Animal monitoring based on IoT technologies," 2018 IoT Vertical and Topical Summit on Agriculture - Tuscany (IOT Tuscany), Tuscany, 2018, pp. $1-5$.

[26] [26] H. Yousif, J. Yuan, R. Kays and Z. He, "Fast humananimal detection from highly cluttered camera-trap images using joint background modeling and deep learning classification," 2017 IEEE International Symposium on Circuits and Systems (ISCAS), Baltimore, MD, 2017, pp. 1-4 